\title{
On Computing Minimal Proper Nullspace Bases with Applications in Fault Detection
}

\author{
Andras Varga \\ German Aerospace Center, DLR - Oberpfaffenhofen \\ Institute of Robotics and Mechatronics \\ D-82234 Wessling, Germany. \\ Andras.Varga@dlr.de
}

Summary. We discuss computationally efficient and numerically reliable algorithms to compute minimal proper nullspace bases of a rational or polynomial matrix. The underlying main computational tool is the orthogonal reduction to a Kronecker-like form of the system matrix of an equivalent descriptor system realization. A new algorithm is proposed to compute a simple minimal proper nullspace basis, starting from a non-simple one. Minimal dynamic cover based computational techniques are used for this purpose. The discussed methods allow a high flexibility in addressing several fault detection related applications.

\section{Introduction}

Consider a $p \times m$ rational matrix $G(\lambda)$, where the indeterminate $\lambda$ is generally a complex variable. If we interpret $G(\lambda)$ as the transfer-function matrix (TFM) of a (generalized) linear time-invariant system, then according to the system type, $\lambda$ is the $s$ variable in the Laplace transform in the case of a continuoustime system or $\lambda$ is the $z$ variable in the $Z$-transform in the case of a discretetime system. This interpretation of $\lambda$ is relevant when system stability aspects are considered.

In this paper we address the following computational problem: For a given $p \times m$ rational or polynomial matrix $G(\lambda)$ with normal rank $r$, determine a $(p-r) \times p$ rational basis matrix $N_{l}(\lambda)$ of the left nullspace of $G(\lambda)$ such that

$$
N_{l}(\lambda) G(\lambda)=0 .
$$

Of special importance are minimal bases having the least achievable McMillan degree. Moreover, depending on the underlying application, further properties may be desirable, as for example, determining $N_{l}(\lambda)$ as a polynomial matrix or as a proper rational matrix with specified poles. 
The rigorous study of polynomial bases started with the theoretical works of Forney [4], and followed by initial algorithmic developments by Kailath [7]. For the computation of a minimal polynomial bases of a polynomial matrix $G(\lambda)$ there are many algorithms, see [1] and the literature cited therein. Two main classes of methods are the resultant methods, which determine the solution by solving directly polynomial equations involving appropriate resultant matrices [1], and pencil methods, which rely on matrix pencil reduction algorithms [2]. While resultant methods can be a real alternative to the unreliable polynomial manipulation based methods proposed in [7], their application to rational matrices defined implicitly via state space system realizations requires, as a supplementary step, bringing the system model into a polynomial representation. This involve factoring $G(\lambda)$ as $G(\lambda)=N(\lambda) M^{-1}(\lambda)$, where $N(\lambda)$ and $M(\lambda)$ are polynomial matrices, and applying the method to $N(\lambda)$. The converse operation (e.g., proper rational factoring of a polynomial matrix) could be also necessary, if the desired basis must be a proper rational basis. Such computational detours are generally considered highly unreliable for TFMs of large scale systems (which usually arise in a state-space form).

The pencil methods works directly on the state space realization of $G(\lambda)$, and are applicable to both polynomial and rational matrices. The main computational tool is the reduction of a matrix pencil to a Kronecker-like form using orthogonal transformations. The left Kronecker structure provides the complete information to compute a polynomial basis via straightforward matrix and polynomial matrix manipulations [2].

For many applications, proper rational bases are required. Such bases can be immediately obtained from polynomial bases. However, to avoid potentially unstable polynomial manipulations, it is of interest to compute proper rational bases directly, without the unnecessary detour of determining first polynomial bases. The theory of proper rational bases has been developed in [13], where the main concepts have been also defined. Of special importance are proper bases which are simple (see the exact definition in the next section), representing a direct generalization of minimal polynomial bases. A first reliable numerical method to compute proper rational bases has been proposed by the author in [20]. This method belongs to the class of pencil methods and its main advantage is that a minimal proper rational basis can be computed by using exclusively orthogonal transformations. Note however, that the resulting basis is generally not simple.

In this paper we extend the algorithm of [20] to compute simple minimal proper rational bases. The new algorithm can be seen as a post-processing method by determining a simple basis starting from a non-simple one. Minimal dynamic covers techniques are used for this purpose. The proposed new algorithm allows to perform easily operations with the resulting basis, which are of importance to solve applications as those encountered in fault detection. For example, computing linear combinations of basis vectors immediately leads to candidate solutions of the fault detection problem with a least order 
detector. Several applications in solving fault detection problems are discussed in a separate section.

\section{Nullspace Bases}

Since polynomial bases represent an important tool in defining the corresponding concepts for the more general rational bases, we will recall shortly some of the main results of [4]. Assume that $N_{l}(\lambda)$ is a polynomial basis of the left nullspace of $G(\lambda)$. Let denote by $n_{i}$, the $i$-th index (or degree), representing the greatest degree of the $i$-th row of $N_{l}(\lambda)$. Then, the order of $N_{l}(\lambda)$ is defined

as $n_{d}=\sum_{i=1}^{p-r} n_{i}$, (i.e., the sum of row degrees). A minimal basis is one which has least order among all polynomial bases. The indices of a minimal basis are called minimal indices. The order of a minimal polynomial basis $N_{l}(\lambda)$ is equal to the McMillan degree of $N_{l}(\lambda)$.

Some properties of a minimal bases are summarized below $[4,7]$ :

Theorem 1. Let $N_{l}(\lambda)$ be a minimal polynomial basis of the left nullspace of $G(\lambda)$ with row indices $n_{i}, i=1, \ldots, p-r$. Then the following holds:

1. The row indices are unique up to permutations (i.e., if $\widetilde{N}_{l}(\lambda)$ is another minimal basis, then $N_{l}(\lambda)$ and $\widetilde{N}_{l}(\lambda)$ have the same minimal indices).

2. The minimal indices are the left Kronecker indices of $G(\lambda)$.

3. $N_{l}(\lambda)$ is irreducible, i.e., has full row rank for all $\lambda \in \mathbb{C}\left(N_{l}(\lambda)\right.$ has no finite or infinite zeros).

4. $N_{l}(\lambda)$ is row reduced, i.e., the leading row coefficient matrix (formed from the coefficients of the highest row degrees) has full row rank.

If $M_{l}(\lambda)$ is a non-singular rational matrix, then $\widetilde{N}_{l}(\lambda):=M_{l}(\lambda) N_{l}(\lambda)$ is also a left nullspace basis. Frequently the matrices $M_{l}(\lambda)$ originate from appropriate left coprime factorizations of an original basis $N_{l}(\lambda)$ in the form

$$
N_{l}(\lambda)=M_{l}(\lambda)^{-1} \widetilde{N}_{l}(\lambda)
$$

where the factors $M_{l}(\lambda)$ and $\widetilde{N}_{l}(\lambda)$ can be choosen to satisfy special requirements (e.g., have only poles in a certain "good" region of the complex plane).

The main advantage of minimal polynomial bases is the possibility to easily build proper minimal rational bases. These are proper rational bases having the least McMillan degree $n_{d}$. A proper minimal rational basis with arbitrary poles can be simply constructed by taking

$$
M_{l}(\lambda)=\operatorname{diag}\left(\frac{1}{m_{1}(\lambda)}, \cdots, \frac{1}{m_{p-r}(\lambda)}\right)
$$

where $m_{i}(\lambda)$ is a polynomial of degree $n_{i}$, and forming $\widetilde{N}_{l}(\lambda):=M_{l}(\lambda) N_{l}(\lambda)$. The resulting basis $\widetilde{N}_{l}(\lambda)$ has the additional property that the order of any 
minimal state space realization of $\widetilde{N}_{l}(\lambda)$ is equal to the sum of orders of the minimal state space realizations of the rows of $\widetilde{N}_{l}(\lambda)$. Furthermore, $D_{l}:=$ $\lim _{\lambda \rightarrow \infty} \widetilde{N}_{l}(\lambda)$ has full row rank. Such a proper basis is termed simple [13] and is the natural counterpart of minimal polynomial basis introduced in [4].

\section{Computation of Minimal Proper Bases}

For the computation of a rational nullspace basis $N_{l}(\lambda)$ a pencil method based on a state space representation of $G(\lambda)$ has been proposed in [20]. In this section we review this algorithm and give some of the properties of the resulting basis. Although minimal, it appears that the resulting minimal basis is not simple. An approach to obtain simple bases is presented in the next section.

The $p \times m$ rational matrix $G(\lambda)$ can be realized as a descriptor system

$$
G(\lambda):=\left[\begin{array}{c|c}
A-\lambda E & B \\
\hline C & D
\end{array}\right]
$$

which is an equivalent notation for

$$
G(\lambda)=C(\lambda E-A)^{-1} B+D
$$

We call this realization irreducible if the pair $(A-\lambda E, B)$ is controllable (i.e., $\operatorname{rank}[A-\lambda E B]=n$ for all $\lambda \subset \mathbb{C})$ and the pair $(A-\lambda E, C)$ is observable (i.e., $\operatorname{rank}\left[A^{T}-\lambda E^{T} C^{T}\right]=n$ for all $\lambda \subset \mathbb{C}$ ) [12], where $n$ is the order of the square matrix $A$.

The computational method described in [20] exploits the simple fact that $N_{l}(\lambda)$ is a left nullspace basis of $G(\lambda)$ if and only if for a suitable $M_{l}(\lambda)$

$$
Y_{l}(\lambda):=\left[M_{l}(\lambda) N_{l}(\lambda)\right]
$$

is a left nullspace basis of the system matrix

$$
S(\lambda)=\left[\begin{array}{cc}
A-\lambda E & B \\
C & D
\end{array}\right]
$$

Thus, to compute $N_{l}(\lambda)$ we can determine first a left nullspace basis $Y_{l}(\lambda)$ for $S(\lambda)$ and then $N_{l}(\lambda)$ simply results as

$$
N_{l}(\lambda)=Y_{l}(\lambda)\left[\begin{array}{c}
0 \\
I_{p}
\end{array}\right]
$$

$Y_{l}(\lambda)$ and thus also $N_{l}(\lambda)$ can be computed by employing linear pencil reduction algorithms based on orthogonal transformations. The main advantage of this approach is that the computation of the nullspace can entirely be done by manipulating state space matrices instead of manipulating polynomial 
models. The resulting nullspace is obtained in a descriptor system representation which can be immediately used in applications. In what follows we give some details of this approach.

Let $Q$ and $Z$ be orthogonal matrices (for instance, determined by using the algorithms of $[2,17])$ such that the transformed pencil $\widetilde{S}(\lambda):=Q S(\lambda) Z$ is in the Kronecker-like staircase form

$$
\widetilde{S}(\lambda)=\left[\begin{array}{cc}
\frac{A_{r}-\lambda E_{r}}{A_{r, l}-\lambda E_{r, l}} \\
\hline 0 & A_{l}-\lambda E_{l} \\
\hline 0 & C_{l}
\end{array}\right]
$$

where the descriptor pair $\left(A_{l}-\lambda E_{l}, C_{l}\right)$ is observable, $E_{l}$ is non-singular, and $A_{r}-\lambda E_{r}$ has full row rank excepting possibly a finite set of values of $\lambda$ (i.e, the invariant zeros of $S(\lambda)$ ). It follows that we can choose the nullspace $\widetilde{Y}_{l}(\lambda)$ of $\widetilde{S}(\lambda)$ in the form

$$
\widetilde{Y}_{l}(\lambda)=\left[0\left|C_{l}\left(\lambda E_{l}-A_{l}\right)^{-1}\right| I\right] .
$$

Then the left nullspace of $S(\lambda)$ is $Y_{l}(\lambda)=\widetilde{Y}_{l}(\lambda) Q$ and can be obtained easily after partitioning suitably $Q$ as

$$
Q=\left[\begin{array}{cc}
\widehat{B}_{r, l} & B_{r, l} \\
\widehat{B}_{l} & B_{l} \\
\widehat{D}_{l} & D_{l}
\end{array}\right]
$$

where the row partitioning corresponds to the column partitioning of $\widetilde{Y}_{l}(\lambda)$ in (7), while the column partitioning corresponds to the row partitioning of $S(\lambda)$ in (5). We obtain

$$
Y_{l}(\lambda)=\left[\begin{array}{c|cc}
A_{l}-\lambda E_{l} & \widehat{B}_{l} B_{l} \\
\hline C_{l} & \widehat{D}_{l} D_{l}
\end{array}\right]
$$

and the nullspace of $G(\lambda)$ is

$$
N_{l}(\lambda)=\left[\begin{array}{c|c}
A_{l}-\lambda E_{l} & B_{l} \\
\hline C_{l} & D_{l}
\end{array}\right]
$$

To obtain this representation of the nullspace basis, we performed exclusively orthogonal transformations on the system matrices. We can prove that all computed matrices are exact for a slightly perturbed original system matrix. It follows that the algorithm to compute the nullspace basis is numerically backward stable.

For an irreducible realization (3) of $G(\lambda)$, the full column rank subpencil $\left[\begin{array}{c}A_{l}-\lambda E_{l} \\ C_{l}\end{array}\right]$ defines also the left Kronecker structure of $G(\lambda)$ [12]. In our case, for $p>m$ this result can be relaxed asking only for controllability 
of the realization (3). Indeed, it can be easily verified that all unobservable eigenvalues of $A-\lambda E$ appear either as invariant zeros or in the right Kronecker structure and thus do not affect the left Kronecker structure of the system pencil $S(\lambda)$. This is not anymore true in the case when the realization (3) is not controllable. In this case, a part of uncontrollable eigenvalues may appear as invariant zeros, while the rest of them enters in $A_{l}-\lambda E_{l}$, thus affecting the left Kronecker structure.

It is possible to obtain the subpencil characterizing the left structure in an observability staircase form

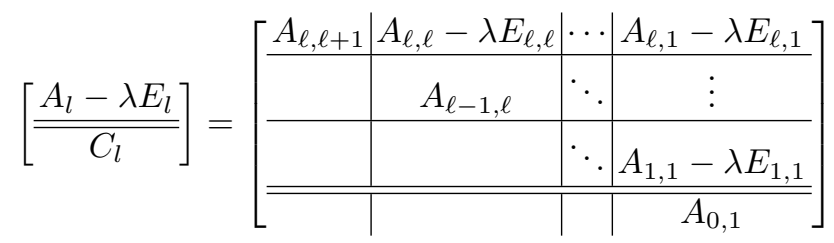

where $A_{i, i+1} \in \mathbb{R}^{\mu_{i} \times \mu_{i+1}}$, with $\mu_{\ell+1}=0$, are full column rank upper triangular matrices, for $i=0, \ldots, \ell$. Note that this form is automatically obtained by using the pencil reduction algorithms described in [2, 17]. The left (or row) Kronecker indices result as follows: there are $\mu_{i-1}-\mu_{i}$ Kronecker blocks of size $i \times(i-1)$, for $i=1, \ldots, \ell+1$. The row dimension of $N_{l}(\lambda)$ (i.e., the number of linearly independent basis vectors) is given by the total number of Kronecker indices, thus $\sum_{i=1}^{\ell+1}\left(\mu_{i-1}-\mu_{i}\right)=\mu_{0}$. Applying standard linear algebra results, it follows that $\mu_{0}:=p-r$.

We give now some properties of the computed rational basis.

Theorem 2. If the realization (3) of $G(\lambda)$ is controllable, then the rational matrix $N_{l}(\lambda)$ defined in (9) is a minimal proper rational basis of the left nullspace of $G(\lambda)$.

Proof. According to the definition of a minimal proper rational basis $[4,13]$, its McMillan degree is given by the sum of row indices of a minimal polynomial basis. The order of the computed basis in (9) is

$$
n_{l}:=\sum_{i=1}^{\ell} \mu_{i}
$$

We have to show that this order is the same as that of an equivalent minimal polynomial basis.

The controllability of the realization (3) ensures that the left Kronecker structure of $G(\lambda)$ and of $S(\lambda)$ are characterized by the same Kronecker indices. Instead of the rational basis $\widetilde{Y}_{l}(\lambda)$ in $(7)$, we can directly compute a minimal polynomial basis of the form

$$
\widehat{Y}_{l}(\lambda)=\left[0 \mid \widehat{N}_{l}(\lambda)\right],
$$


where $\widehat{N}_{l}(\lambda)$ is a minimal polynomial basis for the left nullspace of $\left[\begin{array}{c}A_{l}-\lambda E_{l} \\ C_{l}\end{array}\right]$. For this purpose, we can exploit the staircase form (10). Using the staircase form (10), it is shown in [2] in a dual context that a minimal polynomial basis can be computed by selecting $\mu_{i-1}-\mu_{i}$ polynomial basis vectors of degree $i-1$, for $i=1, \ldots, \ell+1$. This basis can be used to construct a minimal rational basis by making each row proper with appropriate order denominators (as shown in Section 2). The total order of such a basis is

$$
\bar{n}_{l}=\sum_{i=1}^{\ell+1}\left(\mu_{i-1}-\mu_{i}\right)(i-1)
$$

But this is exactly $n_{l}$, since

$$
\begin{aligned}
\bar{n}_{l} & =\sum_{i=1}^{\ell+1} \mu_{i-1}(i-1)-\sum_{i=1}^{\ell+1} \mu_{i}(i-1) \\
& =\sum_{i=1}^{\ell} \mu_{i} i-\sum_{i=1}^{\ell} \mu_{i}(i-1)=\sum_{i=1}^{\ell} \mu_{i}
\end{aligned}
$$

To finish this part of the proof, we need to show additionally that the realization (9) is minimal. The pair $\left(A_{l}-\lambda E_{l}, C_{l}\right)$ is observable, by the construction of the Kronecker-like form (6). To show the pair $\left(A_{l}-\lambda E_{l}, B_{l}\right)$ is controllable, observe that due to the controllability of the pair $(A-\lambda E, B)$, the sub-pencil $[A-\lambda E B]$ has full row rank, and thus the reduced pencil

$$
Q\left[\begin{array}{cc|c}
A-\lambda E & B & 0 \\
C & D & I_{p}
\end{array}\right]\left[\begin{array}{c|c}
Z & 0 \\
0 & I_{p}
\end{array}\right]=\left[\begin{array}{cc|c}
A_{r}-\lambda E_{r} & A_{r, l}-\lambda E_{r, l} & B_{r, l} \\
0 & A_{l}-\lambda E_{l} & B_{l} \\
0 & C_{l} & D_{l}
\end{array}\right]
$$

has full row rank as well. It follows that

$$
\operatorname{rank}\left[A_{l}-\lambda E_{l} B_{l}\right]=n_{l}
$$

and thus the pair $\left(A_{l}-\lambda E_{l}, B_{l}\right)$ is controllable.

Since, we also have that

$$
\operatorname{rank}\left[\begin{array}{cc}
A_{l}-\lambda E_{l} & B_{l} \\
C_{l} & D_{l}
\end{array}\right]=n_{l}+p-r
$$

for all $\lambda$, it follows that $N_{l}(\lambda)$ has no finite or infinite zeros. Thus, $D_{l}$ has full row rank $p-r$ and the computed basis is column reduced at $\lambda=\infty$ [13].

In the case, when the realization of $(3)$ of $G(\lambda)$ is not controllable, the realization of $N_{l}(\lambda)$ is not guaranteed to be controllable. The uncontrollable eigenvalues of $A-\lambda E$ enters partly either as invariant zeros (i.e., part of the sub-pencil $A_{r}-\lambda E_{r}$ ) or are part of the sub-pencil $A_{l}-\lambda E_{l}$. Therefore, in this case, the resulting nullspace basis has not the least possible McMillan degree.

Additionally the following important result holds:

Proposition 1. If the realization (3) of $G(\lambda)$ is controllable, then the realization of $N_{l}(\lambda)$ defined in (9) is maximally controllable. 
Proof. According to a dual formulation of [10], we have to show that for an arbitrary output injection matrix $K$, the pair $\left(A_{l}+K C_{l}-\lambda E_{l}, B_{l}+K D_{l}\right)$ remains controllable. Consider the transformation matrix

$$
U=\left[\begin{array}{ccc}
I & 0 & 0 \\
0 & I & K \\
0 & 0 & I
\end{array}\right]
$$

and compute $\widehat{S}(\lambda):=U Q S(\lambda) Z$, which, due to the particular form of $C_{l}$, is still in the Kronecker-like staircase form

$$
\widehat{S}(\lambda)=\left[\begin{array}{cc}
\frac{A_{r}-\lambda E_{r}}{2} A_{r, l}-\lambda E_{r, l} \\
\hline 0 & A_{l}+K C_{l}-\lambda E_{l} \\
\hline 0 & C_{l}
\end{array}\right]
$$

If we form also

$$
U Q\left[\begin{array}{c}
0 \\
I_{p}
\end{array}\right]=\left[\begin{array}{c}
B_{r, l} \\
B_{l}+K D_{l} \\
D_{l}
\end{array}\right]
$$

we obtain an alternative minimal proper rational basis in the form

$$
\widetilde{N}_{l}(\lambda)=\left[\begin{array}{c|c}
A_{l}+K C_{l}-\lambda E_{l} & B_{l}+K D_{l} \\
\hline C_{l} & D_{l}
\end{array}\right]
$$

We already have proven in Theorem 2 that such a nullspace basis is a minimal realization. Thus, the pair $\left(A_{l}+K C_{l}-\lambda E_{l}, B_{l}+K D_{l}\right)$ is controllable.

Even if the above computed rational basis has the least possible McMillan degree, and thus is minimal, still in general, this basis is not simple. In the next section, we consider a postprocessing approach permitting to obtain a simple basis from a non-simple one.

\section{Computation of Simple Bases}

The most obvious approach to determine a simple minimal proper rational basis has been sketched in Section 2 and consists in computing first a minimal polynomial basis $N_{l}(\lambda)$ and then to determine the rational basis as $\widetilde{N}_{l}(\lambda):=$ $M_{l}(\lambda) N_{l}(\lambda)$, where $M_{l}(\lambda)$ has the form (2).

We discuss shortly the method to compute a polynomial basis proposed in [2]. This method determines first a minimal polynomial basis $W(\lambda)$ for the left nullspace of the sub-pencil $\left[\begin{array}{c}A_{l}-\lambda E_{l} \\ C_{l}\end{array}\right]$ in (6). This computation can be done by fully exploiting the staircase structure (10) of this pencil. The details for a dual algorithm (for right basis) are presented in [2]. The degrees of the resulting left basis vectors are equal to the left Kronecker indices, and this information can be simply read out from the staircase structure. As already 
mentioned, there are $p-r$ basis vectors, of which there are $\mu_{i-1}-\mu_{i}$ vectors of degree $(i-1)$.

The minimal polynomial nullspace basis of $G(\lambda)$ results as

$$
N_{l}(\lambda)=[0 W(\lambda)] Q\left[\begin{array}{c}
0 \\
I_{p}
\end{array}\right]
$$

Note that $W(\lambda)$ and $N_{l}(\lambda)$ have the same row degrees. Furthermore, it is shown in [2] that the resulting $N_{l}(\lambda)$ is row reduced.

The approach to compute a simple proper minimal basis has been sketched in Section 2 and additionally involves to determine $M(\lambda)$ of the form (2), where $m_{i}(\lambda)$ is an arbitrary polynomial of degree $n_{i}$. The resulting simple proper minimal basis is $\widetilde{N}_{l}(\lambda):=M(\lambda) N_{l}(\lambda)$ and has arbitrarily assignable poles. A state-space realization of the resulting basis $\widetilde{N}_{l}(\lambda)$ can be simply built by inspection, exploiting the simpleness property. This realization is obtained by simply stacking $p-r$ minimal realizations of orders $n_{i}, i=1, \ldots, p-r$ of each row of $\widetilde{N}_{l}(\lambda)$. The resulting state matrix has a block diagonal structure. Although simple, this approach is not always well suited for applications (e.g., in fault detection) for reasons which will become apparent in Section 7.

We propose an alternative to this method which is based on minimum cover techniques and, as will be shown later, directly supports the design of least order fault detectors. Consider the proper minimal left nullspace (9) and denote with $c_{l, i}$ and $d_{l, i}$ the $i$-th rows of matrices $C_{l}$ and $D_{l}$, respectively.

Theorem 3. For each $i=1, \ldots, p-r$, let $K_{i}$ be an output injection matrix such that

$$
v_{i}(\lambda):=c_{l, i}\left(\lambda E_{l}-A_{l}-K_{i} C_{l}\right)^{-1}\left(B_{l}+K_{i} D_{l}\right)+d_{l, i}
$$

has the least possible McMillan degree. Then, $\widetilde{N}_{l}(\lambda)$ formed from the $p-r$ rows $v_{i}(\lambda)$ is a simple proper minimal left nullspace basis.

Proof. According to Proposition 1, the realization (9) of $N_{l}(\lambda)$ is maximally controllable, i.e., the pair $\left(A_{l}+K_{i} C_{l}-\lambda E_{l}, B_{l}+K_{i} D_{l}\right)$ is controllable for arbitrary $K_{i}$. Therefore, the maximal order reduction of the McMillan degree of $v_{i}(\lambda)$ can be achieved by making the pair $\left(A_{l}+K_{i} C_{l}-\lambda E_{l}, c_{l, i}\right)$ maximally unobservable via an appropriate choice of $K_{i}$. For each $i=1, \ldots, p-r$, the achievable least McMillan degree of $v_{i}(\lambda)$ is the corresponding minimal index $n_{i}$, representing, in a dual setting, the dimension of the least order controllability subspace of the standard pair $\left(E_{l}^{-T} A_{l}^{T}, E_{l}^{-T} C_{l}^{T}\right)$ containing $\operatorname{span}\left(E_{l}^{-T} c_{l, i}^{T}\right)$. This result is the statement of Lemma 6 in [29]. It is easy to check that $v_{i}(\lambda) G(\lambda)=0$, thus $\widetilde{N}_{l}(\lambda)$ is a left annihilator of $G(\lambda)$. Furthermore, the set of vectors $\left\{v_{1}(\lambda), \ldots, v_{p-r}(\lambda)\right\}$ is linearly independent since the realization of $\widetilde{N}_{l}(\lambda)$ has the same full row rank matrix $D_{l}$ as that of $N_{l}(\lambda)$. It follows that $\widetilde{N}_{l}(\lambda)$ is a proper left nullspace basis of least dimension $\sum_{i=1}^{p-r} n_{i}$, with each row $v_{i}(\lambda)$ of McMillan degree $n_{i}$. It follows that $N_{l}(\lambda)$ is simple. 
The poles of the nullspace basis can be arbitrarily placed by performing left coprime rational factorizations

$$
v_{i}(\lambda)=m_{i}(\lambda)^{-1} \hat{v}_{i}(\lambda)
$$

The basis $\widehat{N}_{l}(\lambda):=\left[\hat{v}_{1}^{T}(\lambda), \ldots, \hat{v}_{p-r}^{T}(\lambda)\right]^{T}$ obtained in this way, can have arbitrarily assigned poles.

Simple bases are the direct correspondents of polynomial bases, and therefore each operation on a polynomial basis has a direct correspondent operation on the corresponding simple rational basis. An important operation (with applications in fault detection) is building linear combinations of basis vectors up to a certain McMillan degree.

Consider the proper left nullspace basis $N_{l}(\lambda)$ constructed in (9). By looking to the details of the resulting staircase form (10) of the pair $\left(A_{l}-\lambda E_{l}, C_{l}\right)$, recall that the full column rank matrices $A_{i-1, i} \in \mathbb{R}^{\mu_{i-1} \times \mu_{i}}$ have the form

$$
A_{i-1, i}=\left[\begin{array}{c}
R_{i-1, i} \\
0
\end{array}\right]
$$

where $R_{i-1, i}$ is an upper-triangular invertible matrix of order $\mu_{i}$. The row dimension $\mu_{i-1}-\mu_{i}$ of the zero block of $A_{i-1, i}$ gives the number of polynomial vectors of degree $i-1$ in a minimal polynomial basis [2, Section 4.6] and thus, also the number of vectors of McMillan degree $i-1$ in a simple basis. It is straightforward to show the following result.

Corollary 1. For a given left nullspace basis $N_{l}(\lambda)$ in the form (9), let $1 \leq$ $i<p-r$ be a given index and let $h$ be $a(p-r)$-dimensional row vector having only the last $i$ components non-zero. Then, a linear combination of the simple basis vectors not exceeding McMillan degree $n_{i}$ can be generated as

$$
v(\lambda):=h C_{l}\left(\lambda E_{l}-A_{l}-K C_{l}\right)^{-1}\left(B_{l}+K D_{l}\right)+h D_{l}
$$

where $K$ is an output injection matrix such that $v(\lambda)$ has the least possible McMillan degree.

This result shows that the determination of a linear combination of vectors of a simple basis up to a given order $n_{i}$ is possible directly from a proper basis determined in the form (9). As it will be shown in the next section, the matrix $K$ together with a minimal realization of $v(\lambda)$ can be computed efficiently using minimal dynamic cover techniques. The same approach can be applied repeatedly to determine the basis vectors $v_{i}(\lambda), i=1, \ldots, p-r$, of a simple basis by using the particular choices $h=e_{i}^{T}$, where $e_{i}$ is the $i$-th column of the $(p-r)$-th order identity matrix.

\section{Minimal dynamic cover techniques}

Let $N_{l}(\lambda)$ be the $(p-r) \times p$ minimal proper left nullspace basis of $G(\lambda)$ constructed in (9). In this section we will address the following computational 
problem encountered when computing simple proper bases or when computing linear combination of basis vectors with least McMillan degree: given a row vector $h$, determine the output injection matrix $K$ such that the vector $v(\lambda)$ in (16) has least McMillan degree. As already mentioned, minimal dynamic cover techniques can be employed to perform this computation.

Computational procedures of minimal dynamic covers are presented in [22] (see also Appendix A). The general idea of the cover algorithms is to perform a similarity transformation on the system matrices in (9) to bring them in a special form which allows to cancel the maximum number of unobservable eigenvalues. In a dual setting, for the so-called Type I dynamic covers [8], two nonsingular transformation matrices $L$ and $V$ result such that

$$
\left[\begin{array}{c}
N_{l}(\lambda) \\
h N_{l}(\lambda)
\end{array}\right]=\left[\begin{array}{c|c}
L\left(A_{l}-\lambda E_{l}\right) V & L B_{l} \\
\hline C_{l} V & D_{l} \\
h C_{l} V & h D_{l}
\end{array}\right]=\left[\begin{array}{cc|c}
\widehat{A}_{11}-\lambda E_{11} & \widehat{A}_{12}-\lambda E_{12} & \widehat{B}_{1} \\
\widehat{A}_{21} & \widehat{A}_{22}-\lambda E_{22} & \widehat{B}_{2} \\
\hline \widehat{C}_{11} & \widehat{C}_{12} & D_{l} \\
0 & \widehat{c}_{22} & h D_{l}
\end{array}\right],
$$

where the pairs $\left(\widehat{A}_{11}-\lambda E_{11}, \widehat{C}_{11}\right)$ and $\left(\widehat{A}_{22}-\lambda E_{22}, \widehat{c}_{22}\right)$ are observable, and the submatrices $\widehat{C}_{11}$ and $\widehat{A}_{21}$ have the particular structure

$$
\left[\begin{array}{l}
\widehat{A}_{21} \\
\widehat{C}_{11}
\end{array}\right]=\left[\begin{array}{ll}
0 & A_{21} \\
0 & C_{11}
\end{array}\right]
$$

with $C_{11}$ having full column rank. By taking

$$
K=V\left[\begin{array}{c}
0 \\
\widehat{K}
\end{array}\right]
$$

with $\widehat{K}$ satisfying $\widehat{K} C_{11}+A_{21}=0$, we annihilate $\widehat{A}_{21}$, and thus make the pair $\left(A_{l}+K C_{l}-\lambda E_{l}, h C_{l}\right)$ maximally unobservable by making all eigenvalues of $\widehat{A}_{11}-\lambda E_{11}$ unobservable. The resulting vector $v(\lambda)$ of least McMillan degree, obtained by deleting the unobservable part, has the minimal state space realization

$$
v(\lambda)=\left[\begin{array}{c|c}
\widehat{A}_{22}+\widehat{K} \widehat{C}_{12}-\lambda E_{22} & \widehat{B}_{2}+\widehat{K} D_{l} \\
\hline \widehat{c}_{22} & h D_{l}
\end{array}\right]
$$

This is also the typical form of achieved realizations for the basis vectors (15) of a simple basis. To obtain the above realization, the computation of the transformation matrices $L$ and $V$ is not necessary, provided all transformations which are performed during the reductions in the minimal cover algorithm are applied to the input matrix $B_{l}$ as well. In Appendix A we present a detailed algorithm for the computation of Type $I$ dynamic covers. 


\section{Computation of proper coprime factorizations}

We present a straightforward application of minimal proper nullspaces in determining proper fractional factorizations of improper rational matrices. This computation is often a preliminary preprocessing step when designing residual generator filters for solving the optimal fault detection problem involving improper systems [25]. Let $G(\lambda)$ be a given $p \times m$ improper rational matrix for which we want to determine a fractional representation in the form

$$
G(\lambda)=M^{-1}(\lambda) N(\lambda),
$$

where both $M(\lambda)$ and $N(\lambda)$ are proper. In applications, the stability of the factors is frequently imposed as an additional requirement. For this computation, state space techniques have been proposed in [18], based on stabilization and pole assignment methods for descriptor systems. We show, that alternatively a conceptually simple and numerically reliable approach can be used to obtain the above factorization.

The relation (19) can be rewritten as

$$
[M(\lambda) N(\lambda)]\left[\begin{array}{l}
G(\lambda) \\
-I_{m}
\end{array}\right]=0 .
$$

It follows that the $p \times(p+m)$ rational matrix $[M(\lambda) N(\lambda)]$ can be determined as a minimal proper left nullspace basis of the full column rank matrix

$$
G_{e}(\lambda)=\left[\begin{array}{c}
G(\lambda) \\
-I_{m}
\end{array}\right]
$$

The invertibility of $M(\lambda)$ is guaranteed by Lemma 2 of [28] by observing that $[M(\lambda) N(\lambda)]$, as a nullspace basis, has full row rank.

Using the state-space realizations based algorithm described in Section 3, we obtain the left nullspace basis $[M(\lambda) N(\lambda)]$ of $G_{e}(\lambda)$ in the form (9) with the matrices $B_{l}$ and $D_{l}$ partitioned accordingly

$$
[M(\lambda) N(\lambda)]=\left[\begin{array}{c|c|c}
A_{l}-\lambda E_{l} & B_{M, l} B_{N, l} \\
\hline C_{l} & D_{M, l} D_{N, l}
\end{array}\right] .
$$

Since $E_{l}$ is invertible, the resulting factors are proper. An important aspect of this simple approach is that the state space realizations (20) of the factors of the proper factorization (19) have been obtained using exclusively orthogonal transformations to reduce the system matrix of $G_{e}(\lambda)$ to a Kronecker-like form as that in (6). This contrasts with the algorithms of [18] which involve also some non-orthogonal manipulations. The stability of the resulting factors can be enforced, using instead (9), a representation of the form (14) for the left nullspace. Here, $K$ is determined to fulfill the stability requirements. 


\section{Operations involving nullspace bases}

Assume that besides the $p \times m$ rational matrix $G(\lambda)$, we have given also a $p \times q$ rational matrix $F(\lambda)$, and the compound matrix $[G(\lambda) F(\lambda)]$ has the state space realization

$$
[G(\lambda) F(\lambda)]=\left[\begin{array}{c|cc}
A-\lambda E & B B_{f} \\
\hline C & D D_{f}
\end{array}\right] .
$$

Observe that the realizations of $G(\lambda)$ and $F(\lambda)$ share the same state, descriptor and output matrices $A, E$, and $C$, respectively. Let $N_{l}(\lambda)$ be a proper left nullspace basis of $G(\lambda)$ which can be non-simple in the form in (9) or a simple basis formed with vectors of the form (15). In several applications, besides the computation of the nullspace basis, operations with the basis matrix are necessary. For example, the left multiplications $N_{l}(\lambda) F(\lambda)$ or $\widetilde{N}_{l}(\lambda) F(\lambda)$, where $N_{l}(\lambda)=M_{l}^{-1}(\lambda) \widetilde{N}_{l}(\lambda)$ is a left coprime factorization, are often necessary in fault detection applications. Important are also operations involving a linear combination of the basis vectors, i.e., the computation of $v(\lambda) F(\lambda)$, where $v(\lambda)$ has the form (16) or is in a minimal form (18) as resulted from the application of the minimal cover algorithm. This last operation is also important when computing $N_{l}(\lambda) F(\lambda)$ with $N_{l}(\lambda)$ a simple proper left nullspace basis formed from row vectors of the form (15).

The determination of state space realizations of products like $N_{l}(\lambda) F(\lambda)$, $\widetilde{N}_{l}(\lambda) F(\lambda)$ or $v(\lambda) F(\lambda)$ can be done by computing minimal realizations of the state space realizations of these rational matrix products. The computation of a minimal realization relies on numerically stable algorithms for standard or descriptor systems as those proposed in [14, 15]. However, these algorithms depend on intermediary rank determinations and thus can produce results which critically depend on the choice of threshold values used to detect zero elements. Since it is always questionable that the resulting order is the correct one, this computational approach can be categorized as a difficult numerical computation. Alternative ways relying on balancing related model reduction are primarily intended for standard stable systems. The application of this approach in the case when $F(\lambda)$ is unstable or not proper leads to other types of numerical difficulties. For example, by assuming that the unstable/improper part cancels completely out, a preliminary spectral splitting of eigenvalues must be performed first, which is often associated with unnecessary accuracy losses. For polynomial nullspace bases the only alternative to the above approach is to manipulate polynomial matrices. However, as already mentioned, in some applications this leads to unavoidable detours (state-space to polynomial model conversions) which involve delicate rank decisions as well.

In what follows, we show that all these numerical difficulties to evaluate the above products can be completely avoided and explicit state space realizations for these products can be obtained as a natural byproduct of the nullspace computation procedure. An important aspect of the developed explicit realizations is that both $N_{l}(\lambda)$ and $N_{l}(\lambda) F(\lambda)$ share the same state, 
descriptor and output matrices. Thus, the developed formulas are also useful for performing nullspace updating and two important applications of this techniques in the context of fault detection are presented in the next section.

\subsection{Left Multiplication with a Non-simple Basis}

Let $N_{l}(\lambda)$ be a proper left nullspace basis of $G(\lambda)$ computed in the form (9) and let $Y_{l}(\lambda)$ be the left nulspace basis of $S(\lambda)$ in (4). It is easy to show that

$$
Y_{l}(\lambda)\left[\begin{array}{c|c}
A-\lambda E & B_{f} \\
C & D_{f}
\end{array}\right]=\left[0 \mid N_{l}(\lambda) F(\lambda)\right]
$$

and thus

$$
N_{l}(\lambda) F(\lambda)=Y_{l}(\lambda)\left[\begin{array}{c}
B_{f} \\
D_{f}
\end{array}\right]=\widetilde{Y}_{l}(\lambda) Q\left[\begin{array}{c}
B_{f} \\
D_{f}
\end{array}\right],
$$

where $Q$ is the orthogonal transformation matrix used in computing the Kronecker-like form (6) and $\widetilde{Y}_{l}(\lambda)$ is defined in (7). We compute now

$$
Q\left[\begin{array}{c}
B_{f} \\
D_{f}
\end{array}\right]=\left[\begin{array}{c}
* \\
\widetilde{\widetilde{B}_{f}} \\
\hline \widetilde{D}_{f}
\end{array}\right],
$$

where the row partitioning of the right hand side corresponds to the column partitioning of $\widetilde{Y}_{l}(\lambda)$ in $(7)$. The realization of $N_{l}(\lambda) F(\lambda)$ results as

$$
N_{l}(\lambda) F(\lambda)=\left[\begin{array}{c|c}
A_{l}-\lambda E_{l} & \widetilde{B}_{f} \\
\hline C_{l} & \widetilde{D}_{f}
\end{array}\right] .
$$

Note that to compute this realization, only orthogonal transformations have been employed.

In assessing the properties of the resulting realization (23), two aspects are relevant. The realizations of $N_{l}(\lambda)$ and $N_{l}(\lambda) F(\lambda)$ are observable since they share the same $A_{l}, E_{l}$ and $C_{l}$ matrices. However, the realization in (23) may not be minimal, because its controllability also depends on the involved $B_{f}$ and $D_{f}$. The second aspect concerns the minimality of the proper left nullspace basis $N_{l}(\lambda)$ itself. When computing $N_{l}(\lambda)$, we can freely assume that that the overall realization of $[G(\lambda) F(\lambda)]$ is irreducible. According to Proposition 2, to obtain a minimal proper basis for the left nullspace of $G(\lambda)$ using the proposed rational nullspace procedure, the corresponding realization in (21) must be controllable. Although this condition is usually fulfilled in fault detection applications (see Section 8.1), still the realization of $G(\lambda)$ can be in general uncontrollable, and therefore the resulting left nullspace basis $N_{l}(\lambda)$ may not have the least possible McMillan degree. This can be also the case for the resulting realization (23) of $N_{l}(\lambda) F(\lambda)$. These two aspects 
are the reasons why the order of the resulting realization of $N_{l}(\lambda) F(\lambda)$ in (23) may exceed the least possible one (which can be obtained by working exclusively with minimal realizations and employing the already mentioned minimal realization techniques).

\subsection{Left Coprime Factorization}

Assume $N_{l}(\lambda)$ be the left nullspace basis in (9). In several applications, this rational basis must be stable, that is, to have in a continuous-time setting only poles with negative real parts, or in a discrete-time setting poles inside the unit circle of the complex plane. As already mentioned, instead $N_{l}(\lambda)$ we can freely use as left nullspace basis $\widetilde{N}_{l}(\lambda)$, the denominator factor of the left fractional representation

$$
N_{l}(\lambda)=M_{l}^{-1}(\lambda) \tilde{N}_{l}(\lambda)
$$

where $M_{l}(\lambda)$ and $\widetilde{N}_{l}(\lambda)$ are rational matrices with poles in appropriate stability domains. A state space realization of $\left[\widetilde{N}_{l}(\lambda) M_{l}(\lambda)\right]$ is given by well known formulas [32]

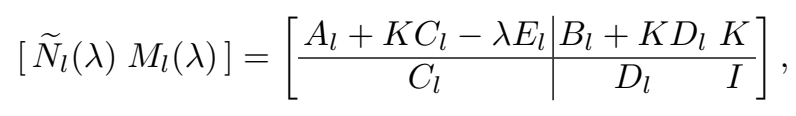

where $K$ is an appropriate output injection matrix which assigns the eigenvalues of $A_{l}+K C_{l}-\lambda E_{l}$ in desired positions or in a suitable stability domain. Recall that this is always possible, since the pair $\left(A_{l}-\lambda E_{l}, C_{l}\right)$ is observable. Numerically reliable algorithms to determine a suitable $K$ can be used based on pole assignment or stabilization techniques [16]. Alternatively, recursive factorization techniques as those proposed in [18] can be employed.

With $U$ of the form (12), we can compute

$$
U Q\left[\begin{array}{c}
B_{f} \\
D_{f}
\end{array}\right]=\left[\begin{array}{c}
* \\
\widetilde{B}_{f}+K \widetilde{D}_{f} \\
\widetilde{D}_{f}
\end{array}\right]
$$

and in a completely similar way as in the previous subsection, we can obtain the realization of $N_{l}(\lambda) F(\lambda)$ as

$$
\tilde{N}_{l}(\lambda) F(\lambda)=\left[\begin{array}{c|c}
A_{l}+K C_{l}-\lambda E_{l} & \widetilde{B}_{f}+K \widetilde{D}_{f} \\
\hline C_{l} & \widetilde{D}_{f}
\end{array}\right] .
$$

When employing the algorithms in [18], a supplementary orthogonal similarity transformation is also implicitly applied to the resulting system matrices, such that the resulting pencil $A_{l}+K C_{l}-\lambda E_{l}$ is in a quasi-triangular (generalized real Schur) form. This computation can be seamlessly integrated 
into the evaluation of $\widetilde{N}_{l}(\lambda) F(\lambda)$ if we perform the left coprime factorization algorithm directly to the compound matrix realization

$$
\left[N_{l}(\lambda) N_{l}(\lambda) F(\lambda)\right]=\left[\begin{array}{c|cc}
A_{l}-\lambda E_{l} & B_{l} & \widetilde{B}_{f} \\
\hline C_{l} & D_{l} & \widetilde{D}_{f}
\end{array}\right] .
$$

In the case of a simple basis, this technique can be employed by considering fractional representations of the form $(24)$ with $M_{l}(\lambda)$ diagonal. In this case the same algorithm can be applied to each row of $\left[N_{l}(\lambda) N_{l}(\lambda) F(\lambda)\right]$, by exploiting the block-diagonal structure of the underlying $A_{l}-\lambda E_{l}$ to increase the efficiency of computations.

\subsection{Left Multiplication with a Simple Nullspace Basis}

We show first how to compute $v(\lambda) F(\lambda)$, where $v(\lambda)$ is given in (16). The same formula applies for a vector of the form (15), with obvious replacements. By observing that $v(\lambda)=h \widetilde{N}_{l}(\lambda)$ with $\widetilde{N}_{l}(\lambda)$ having the form (25), it follows immediately

$$
v(\lambda) F(\lambda)=\left[\begin{array}{c|c}
A_{l}+K C_{l}-\lambda E_{l} & \widetilde{B}_{f}+K \widetilde{D}_{f} \\
\hline h C_{l} & h \widetilde{D}_{f}
\end{array}\right] .
$$

In the case when $K$ has been obtained from the cover algorithm, the minimal realization of $v(\lambda)$ (after eliminating the unobservable part) is given in (18). The corresponding realization of $v(\lambda) F(\lambda)$ is

$$
v(\lambda) F(\lambda)=\left[\begin{array}{c|c}
\widehat{A}_{22}+\widehat{K} \widehat{C}_{12}-\lambda E_{22} & \widehat{B}_{f, 2}+\widehat{K} \widetilde{D}_{f} \\
\hline \widehat{c}_{22} & h \widetilde{D}_{f}
\end{array}\right]
$$

where we used

$$
L \widetilde{B}_{f}=\left[\begin{array}{c}
\widehat{B}_{f, 1} \\
\widehat{B}_{f, 2}
\end{array}\right]
$$

with the transformation matrix $L$ employed in (17) and the row partition corresponding to that of the input matrix $L B_{l}$ in (17). Note that the explicit computation of transformation matrix $L$ is not necessary, because the minimal realization of the product $v(\lambda) F(\lambda)$ can be directly obtained by applying the performed transformations in the minimal cover algorithm (see Appendix A) to the input matrices of the compound realization

$$
\left[\begin{array}{cc}
N_{l}(\lambda) & N_{l}(\lambda) F(\lambda) \\
h N_{l}(\lambda) & h N_{l}(\lambda) F(\lambda)
\end{array}\right]=\left[\begin{array}{c|cc}
A_{l}-\lambda E_{l} & B_{l} & \widetilde{B}_{f} \\
\hline C_{l} & D_{l} & \widetilde{D}_{f} \\
h C_{l} & h D_{l} & h \widetilde{D}_{f}
\end{array}\right]
$$

To compute the products $v_{i}(\lambda) F(\lambda)$, for $i=1, \ldots, p-r$, the same approach can be used taking into account the particular form of $h=e_{i}^{T}$. 
If $N_{l}(\lambda)$ is a simple basis formed from row vectors of the form (16), then the resulting state space realization for $N_{l}(\lambda) F(\lambda)$ is obtained by stacking the realizations of $v_{i}(\lambda) F(\lambda)$ for $i=1, \ldots, p-r$. Also in this case, $N_{l}(\lambda)$ and $N_{l}(\lambda) F(\lambda)$ will share the same state, descriptor and output matrices (i.e., $A_{l}$, $E_{l}, C_{l}$ ), and the pole pencil $A_{l}-\lambda E_{l}$ will have a block diagonal form, where the dimensions of the diagonal blocks are the minimal indices $n_{i}$.

\section{Applications to fault detection}

We consider the linear time-invariant system described by input-output relations of the form

$$
\mathbf{y}(\lambda)=G_{u}(\lambda) \mathbf{u}(\lambda)+G_{d}(\lambda) \mathbf{d}(\lambda)+G_{f}(\lambda) \mathbf{f}(\lambda),
$$

where $\mathbf{y}(\lambda), \mathbf{u}(\lambda), \mathbf{d}(\lambda)$, and $\mathbf{f}(\lambda)$ are Laplace- or Z-transformed vectors of the $p$-dimensional system output vector $y(t), m_{u}$-dimensional control input vector $u(t), m_{d}$-dimensional disturbance vector $d(t)$, and $m_{f}$-dimensional fault signal vector $f(t)$, respectively, and where $G_{u}(\lambda), G_{d}(\lambda)$ and $G_{f}(\lambda)$ are the TFMs from the control inputs to outputs, disturbances to outputs, and fault signals to outputs, respectively.

In what follows we will address three applications of the techniques developed in the previous sections.

\subsection{Solving fault detection problems with least order detectors}

The following is the standard formulation of the Fault Detection Problem (FDP): Determine a proper and stable linear residual generator (or fault detector) having the general form

$$
\mathbf{r}(\lambda)=R(\lambda)\left[\begin{array}{l}
\mathbf{y}(\lambda) \\
\mathbf{u}(\lambda)
\end{array}\right]
$$

such that: $(i) r(t)=0$ when $f(t)=0$ for all $u(t)$ and $d(t)$; and $(i i) r(t) \neq 0$ when $f_{i}(t) \neq 0$, for $i=1, \ldots, m_{f}$. Besides the above requirements it is often required for practical use that the TFM of the detector $R(\lambda)$ has the least possible McMillan degree. Note that as fault detector, we can always choose $R(\lambda)$ as a rational row vector.

The requirements $(i)$ and $(i i)$ can be easily transcribed into equivalent algebraic conditions. The (decoupling) condition $(i)$ is equivalent to

$$
R(\lambda) G(\lambda)=0
$$

where

$$
G(\lambda)=\left[\begin{array}{cc}
G_{u}(\lambda) & G_{d}(\lambda) \\
I_{m_{u}} & 0
\end{array}\right]
$$


while the (detectability) condition (ii) is equivalent to

$$
R_{f_{i}}(\lambda) \neq 0, \quad i=1, \ldots, m_{f},
$$

where $R_{f_{i}}(\lambda)$ is the $i$-th column of

$$
R_{f}(\lambda):=R(\lambda)\left[\begin{array}{c}
G_{f}(\lambda) \\
0
\end{array}\right]
$$

Let $G_{f_{i}}(\lambda)$ be the $i$-th column of $G_{f}(\lambda)$. A necessary and sufficient condition for the existence of a solution is the following one [3, 11]:

Theorem 4. For the system (26) the FDP is solvable if and only if

$$
\operatorname{rank}\left[G_{d}(\lambda) G_{f_{i}}(\lambda)\right]>\operatorname{rank} G_{d}(\lambda), \quad i=1, \ldots, m_{f}
$$

From (28) it appears that $R(\lambda)$ is a left annihilator of $G(\lambda)$, thus one possibility to determine $R(\lambda)$ is to compute first a left minimal basis $N_{l}(\lambda)$ for the left nullspace of $G(\lambda)$, and then to build a stable scalar output detector as

$$
R(\lambda)=h(\lambda) N_{l}(\lambda),
$$

representing a linear combination of the rows of $N_{l}(\lambda)$, such that conditions (30) are fulfilled. The above expression represents a parametrization of all possible scalar output fault detectors and is the basis of the so-called nullspace methods.

The first nullspace method to design residual generators for fault detection has been formally introduced in [5], where a polynomial basis based approach was used. This approach has been later extended to rational bases in [20, 24]. The main advantage of the nullspace approach is that the least order design aspect is naturally present in the formulation of the method. In a recent survey [26], it was shown that the nullspace method also provides a unifying design paradigm for most of existing approaches, which can be interpreted as special cases of this method.

Consider a descriptor state space realization of (26)

$$
\begin{aligned}
E \lambda x(t) & =A x(t)+B_{u} u(t)+B_{d} d(t)+B_{f} f(t) \\
y(t) & =C x(t)+D_{u} u(t)+D_{d} d(t)+D_{f} f(t)
\end{aligned},
$$

where $\lambda x(t)=\dot{x}(t)$ or $\lambda x(t)=x(t+1)$ depending on the type of the system, continuous or discrete, respectively. For convenience, in what follows we assume the pair $(A-\lambda E, C)$ is observable and the pair $\left(A-\lambda E,\left[B_{u} B_{d}\right]\right)$ is controllable. This latter condition is typically fulfilled when considering actuator and sensor faults. In this case, $B_{f}$ has partly the same columns as $B_{u}$ (in the case of actuator faults) or zero columns (in the case of sensor faults).

$G(\lambda)$ defined in (29) has the irreducible realization 


$$
G(\lambda)=\left[\begin{array}{c|cc}
A-\lambda E & B_{u} & B_{d} \\
\hline C & D_{u} & D_{d} \\
0 & I_{m_{u}} & 0
\end{array}\right]
$$

Using the method described in Section 3, we compute first a minimal proper left nullspace basis $N_{l}(\lambda)$ of $G(\lambda)$. The state space realization of the $(p-r) \times$ $\left(p+m_{u}\right)$ TFM $N_{l}(\lambda)$ is given by $(9)$, where $r$ is the rank of $G_{d}(\lambda)$.

To check the existence conditions of Theorem 4, we use (23) to compute

$$
N_{f}(\lambda):=N_{l}(\lambda)\left[\begin{array}{c|c}
G_{f}(\lambda) \\
0
\end{array}\right]=\left[\begin{array}{c|c}
A_{l}-\lambda E_{l} & \widetilde{B}_{f} \\
\hline C_{l} & \widetilde{D}_{f}
\end{array}\right]
$$

where

$$
Q\left[\begin{array}{c}
B_{f} \\
D_{f} \\
0
\end{array}\right]=\left[\begin{array}{c}
* \\
\widetilde{B}_{f} \\
\widetilde{D}_{f}
\end{array}\right]
$$

Since the pair $\left(A_{l}-\lambda E_{l}, C_{l}\right)$ is observable, checking the condition (30) is equivalent to verify that

$$
\left[\begin{array}{c}
\widetilde{B}_{f_{i}} \\
\widetilde{D}_{f_{i}}
\end{array}\right] \neq 0, \quad i=1, \ldots, m_{f}
$$

where $\widetilde{B}_{f_{i}}$ and $\widetilde{D}_{f_{i}}$ denote the $i$-th columns of $\widetilde{B}_{f}$ and $\widetilde{D}_{f}$, respectively.

To address the determination of least order scalar output detectors, we can compute linear combinations of the basis vectors of a simple proper basis of increasing McMillan degrees and check the detectability condition (30) for the resulting vectors (seen as candidate detectors). According to Corollary 1 , this comes down to choose an appropriate $h$ and obtain the corresponding $K$ such that the row vector $v(\lambda)=h \widetilde{N}_{l}(\lambda)$ in (16) has the least possible McMillan order. Note that in general, with a randomly generated $h$, one achieves a detector whose order is $\ell$, the maximum degree of a minimal polynomial basis. Recall that $\ell$ is the number of nonzero subdiagonal blocks in the Kroneckerlike form (10) and represents the observability index of the observable pair $\left(A_{l}-\lambda E_{l}, C_{l}\right)$. In the case when no disturbance inputs are present, this is a well know result in designing functional observers [9].

Lower orders detectors can be obtained using particular choices of the row vector $h$. Using Corollary 1, by choosing $h$ with only the trailing $i$ components nonzero, the corresponding linear combination of $i$ basis vectors has McMillan degree $n_{i}$. A systematic search can be performed by generating successive candidates for $h$ with increasing number of nonzero elements and checking for the resulting residual generator the conditions (30). The resulting detectors have non-decreasing orders and thus the first detector satisfying these conditions represents a satisfactory least order design. To speed up the selection, the choice of the nonzero components of $h$ can be done such that for a given tentative order $n_{i}$ a combination of all $\mu_{0}-\mu_{i}$ intervening vectors of order less 
than or equal to $n_{i}$ is built. In this way, repeated checks for the same order are avoided and the search is terminated in at most $\ell$ steps.

For the final design, the resulting dynamics of the detector can be arbitrarily assigned by choosing the detector in the form

$$
R(\lambda)=m(\lambda) h \widetilde{N}_{l}(\lambda),
$$

where $m(\lambda)$ is an appropriate scalar transfer function. Note that the resulting least order at previous step is preserved provided $m(\lambda)$ is computed using coprime factorization techniques [18].

\subsection{Solving Fault Isolation Problems}

The more advanced functionality of fault isolation (i.e., exact location of faults) can be often achieved by designing a bank of fault detectors [6] or by direct design of fault isolation filters [21]. Designing detectors which are sensitive to some faults and insensitive to others can be reformulated as a standard FDP, by formally redefining the faults to be rejected in the residual as fictive disturbances.

Let $R(\lambda)$ be a given detector and let $R_{f}(\lambda)$ be the corresponding fault-toresidual TFM in (31). We define the fault signature matrix $S$, with the $(i, j)$ entry $S_{i j}$ given by

$S_{i j}=1$, if the $(i, j)$ entry of $R_{f}(\lambda)$ is nonzero;

$S_{i j}=0$, if the $(i, j)$ entry of $R_{f}(\lambda)$ is zero.

If $S_{i j}=1$, then we say that the fault $j$ is detected in residual $i$ and if $S_{i j}=0$, then the fault $j$ is decoupled (not detected) in residual $i$.

The following fault detection and isolation problem (FDIP) can be now formulated: Given a $q \times m_{f}$ fault signature matrix $S$ determine a bank of $q$ stable and proper scalar output residual generator filters

$$
\mathbf{r}_{i}(\lambda)=R^{i}(\lambda)\left[\begin{array}{l}
\mathbf{y}(\lambda) \\
\mathbf{u}(\lambda)
\end{array}\right], i=1, \ldots, q
$$

such that, for all $u(t)$ and $d(t)$ we have:

(i) $r_{i}(t)=0$ when $f_{j}(t)=0, \forall j$ with $S_{i j} \neq 0$;

(ii) $r_{i}(t) \neq 0$ when $f_{j}(t) \neq 0, \forall j$ with $S_{i j} \neq 0$.

In this formulation of the FDIP, each scalar output detector $R^{i}(\lambda)$ achieves the fault signature specified by the $i$-th row of the desired fault signature matrix $S$. The resulting global detector corresponding to this $S$ can be assembled as

$$
R(\lambda)=\left[\begin{array}{c}
R^{1}(\lambda) \\
\vdots \\
R^{q}(\lambda)
\end{array}\right]
$$

Let $S$ be a given $q \times m_{f}$ fault signature matrix and denote by $\bar{G}_{f}^{i}(\lambda)$ the matrix formed from the columns of $G_{f}(\lambda)$ whose column indices $j$ correspond 
to zero elements in row $i$ of $S$. The solvability conditions of the FDIP build up from the solvability of $q$ individual FDPs.

Theorem 5. For the system (26) the FDIP with the given fault signature matrix $S$ is solvable if and only if for each $i=1, \ldots, q$, we have

$$
\operatorname{rank}\left[G_{d}(\lambda) \bar{G}_{f}^{i}(\lambda) G_{f_{j}}(\lambda)\right]>\operatorname{rank}\left[G_{d}(\lambda) \bar{G}_{f}^{i}(\lambda)\right]
$$

for all $j$ such that $S_{i j} \neq 0$.

The standard approach to determine $R(\lambda)$ is to design for each row $i$ of the fault signature matrix $S$, a detector $R^{i}(\lambda)$ which generates the $i$-th residual signal $r_{i}(t)$, and thus represents the $i$-th row of $R(\lambda)$. For this purpose, the nullspace method of the previous subsection can be applied with $G(\lambda)$ in (29) replaced by

$$
G(\lambda)=\left[\begin{array}{ccc}
G_{u}(\lambda) & G_{d}(\lambda) & \bar{G}_{f}^{i}(\lambda) \\
I_{m_{u}} & 0 & 0
\end{array}\right]
$$

and with a redefined fault to output TFM $\widetilde{G}_{f}^{i}(\lambda)$, formed from the columns of $G_{f}(\lambda)$ whose indices $j$ correspond to $S_{i j} \neq 0$. The McMillan degree of the global detector (37) is bounded by the sum of the McMillan degrees of the component detectors. Note that this upper bound can be effectively achieved, for example, by choosing mutually different poles for the individual detectors. It is to be expected that lower orders result when the scalar detectors share their poles.

Using the least order design techniques described in this paper, for each row of $S$ we can design a scalar output detector of least McMillan degree. However, even if each detector has the least possible order, there is generally no guarantee that the resulting order of $R(\lambda)$ is also the least possible one. To the best of our knowledge, the determination of a detector of least global McMillan degree for a given specification $S$ is still an open problem. A solution to this problem has been recently suggested in [24] and involves a post processing step as follows.

Assume that the resulting least order scalar detector $R^{i}(\lambda)$ has McMillan degree $\nu_{i}$, for $i=1, \ldots, q$. We can easily ensure that for $\nu_{i} \leq \nu_{j}$, the poles of $R^{i}(\lambda)$ are among the poles of $R^{j}(\lambda)$. The resulting global detector $R(\lambda)$ according to (37) has a McMillan degree which is conjectured in [24] to be the least possible one.

We describe now an improved approach in two steps to design a bank of detectors, which for larger values of $q$, is potentially more efficient than the above standard approach. In a first step, we can reduce the complexity of the original problem by decoupling the influences of disturbances and control inputs on the residuals. In a second stage, a residual generation filter is determined for a system without control and disturbance inputs which achieves the desired fault signature. 
Let $N_{l}(\lambda)$ be a minimal left nullspace basis for $G(\lambda)$ defined in (29) and define a new system without control and disturbance inputs as

$$
\widetilde{\mathbf{y}}(\lambda):=N_{f}(\lambda) \mathbf{f}(\lambda),
$$

where

$$
N_{f}(\lambda):=N_{l}(\lambda)\left[\begin{array}{c}
G_{f}(\lambda) \\
0
\end{array}\right] .
$$

The system (39) has generally a reduced McMillan degree and also a reduced number of outputs $p-r$, where $r$ is the normal rank of $G_{d}(\lambda)$. The state space realization of the resulting $N_{f}(\lambda)$ is given in (35). Observe that $N_{l}(\lambda)$ and $N_{f}(\lambda)$ share the same state, descriptor and output matrices in their realizations.

For the reduced system (39) with TFM $N_{f}(\lambda)$ we can determine, using the standard approach, a bank of $q$ scalar output least order detectors of the form

$$
\mathbf{r}_{i}(\lambda)=\widetilde{R}^{i}(\lambda) \widetilde{\mathbf{y}}(\lambda), i=1, \ldots, q
$$

such that the same conditions are fulfilled as for the original FDIP. The TFM of the final detector can be assembled as

$$
R(\lambda)=\left[\begin{array}{c}
\widetilde{R}^{1}(\lambda) \\
\vdots \\
\widetilde{R}^{q}(\lambda)
\end{array}\right] N_{l}(\lambda)
$$

Comparing (42) and (37) we have

$$
R^{i}(\lambda)=\widetilde{R}^{i}(\lambda) N_{l}(\lambda),
$$

which can be also interpreted as an updating formula of a preliminary (incomplete) design. The resulting order of the $i$-th detector is the same as before, but this two steps approach has the advantage that the nullspace computation and the associated least order design involve systems of reduced orders (in the sizes of state, input and output vectors). The realization of $R^{i}(\lambda)$ can be obtained using the explicit formulas derived in Subsection 7.1.

The improved approach relies on the detector updating techniques which can be easily performed using the explicit realizations of the underlying products. This can be seen as a major advantage of rational nullspace based methods in contrast to polynomial nullspace based computations.

The above procedure has been used for the example studied in [31, Table 2], where a $18 \times 9$ fault signature matrix $S$ served as specification. The underlying system has order 4 . Each line of $S$ can be realized by a detector of order 1 or 2 with eigenvalues $\{-1\}$ or $\{-1,-2\}$. The sum of orders of the resulting individual detectors is 32 , but the resulting global detector $R(\lambda)$ has McMillan degree 6. Recall that the "least order" detector computed in [31] has order 14 . 


\subsection{The computation of achievable fault signatures}

An aspect apparently not addressed until recently in the literature is the generation of the achievable complete fault signatures for a FDIP. Traditionally this aspect is addressed by trying to design a bank of detectors to achieve a desired specification matrix $S$. The specification is achievable if the design was successful. However, it is possible to generate systematically all possible specifications using an exhaustive search. For this purpose, a recursive procedure can be devised which has as inputs the $p \times m$ and $p \times m_{f}$ TFMs $G(\lambda)$ and $F(\lambda)$ and as output the corresponding signature matrix $S$. If we denote this procedure as FDISPEC $(G, F)$, then the fault signature matrix for the system (26) can be computed as

$$
S=F D I S P E C\left(\left[\begin{array}{cc}
G_{u} & G_{d} \\
I_{m_{u}} & 0
\end{array}\right],\left[\begin{array}{c}
G_{f} \\
0
\end{array}\right]\right)
$$

Procedure $S=F D I S P E C(G, F)$

1. Compute a left nullspace basis $N_{l}(\lambda)$ of $G(\lambda)$; exit with empty $S$ if $N_{l}(\lambda)$ is empty.

2. Compute $N_{f}(\lambda)=N_{l}(\lambda) F(\lambda)$.

3. Compute the signature matrix $S$ of $N_{f}(\lambda)$; exit if $S$ is a row vector.

4. For $i=1, \ldots, m_{f}$

4.1 Form $\widetilde{G}_{i}(\lambda)$ as column $i$ of $N_{f}(\lambda)$.

4.2 Form $\widetilde{F}_{i}(\lambda)$ from the columns $1, \ldots, i-1, i+1, \ldots, m_{f}$ of $N_{f}(\lambda)$.

4.3 Call $\widetilde{S}=F D I S P E C\left(\widetilde{G}_{i}, \widetilde{F}_{i}\right)$.

4.4 Partition $\widetilde{S}=\left[\widetilde{S}_{1} \widetilde{S}_{2}\right]$ such that $\widetilde{S}_{1}$ has $i-1$ columns.

4.5 Define $\widehat{S}=\left[\begin{array}{lll}\widetilde{S}_{1} & 0 & \widetilde{S}_{2}\end{array}\right]$ and update $S \leftarrow\left[\begin{array}{l}S \\ \widehat{S}\end{array}\right]$.

As it can be observed, the efficient implementation of this procedure heavily benefits of the state space updating techniques developed in Subsection 7.1. This confers an increased efficiency during the recursive calls, because the dimensions of the systems are decreasing during a full recursion. The current recursion is broken each time an empty nullspace is encountered or when the last possible recursion level has been attained (i.e., $S$ at Step 3 is a row vector). The computation of structural information at Step 3 involves checking for zero columns in the input and feedthrough matrices $\widetilde{B}_{f}$ and $\widetilde{D}_{f}$ of the realization of $N_{f}(\lambda)$ in (23). Note that the whole recursive computations can be performed by using exclusively orthogonal transformations.

The above procedure can be easily implemented such that it performs the minimum number of nullspace computations and updating. The resulting fault signature matrix $S$ is obtained by stacking row-wise the matrices $S_{i}, i=$ $1, \ldots, k$ computed at different recursion levels, where $k$ denotes the number of calls of the recursive procedure. This number is given by the combinatorial formula 


$$
k=\sum_{i=0}^{i_{\max }}\left(\begin{array}{c}
m_{f} \\
i
\end{array}\right),
$$

where $i_{\max }=\min \left(m_{f}, p-r\right)-1$ and $r$ is the rank of the initial $G(\lambda)$. As can be observed, $k$ depends of the number of basis vectors $p-r$ and the number of faults $m_{f}$, and, although the number of distinct specifications can be relatively low, still $k$ can be a large number. For the already mentioned problem in [31], $k=1+m_{f}+m_{f}\left(m_{f}-1\right) / 2=37$, but only 18 of the determined specifications are distinct. A detailed account of the computational aspects of the procedure FDISPEC is done in [27].

\section{Conclusions}

In this paper we presented an overview of computational techniques to determine rational nullspace bases of rational or polynomial matrices. Simple proper rational bases are the direct correspondents of the polynomial bases and can be computed using the proposed numerical algorithms based on minimal cover techniques. Having in mind potential applications, we also developed explicit realizations for several operation with nullspace bases or with a linear combination of vectors of a nullspace basis. The computational techniques presented in this paper have been implemented as robust numerical software which is now part of a Descriptor System Toolbox for Matlab developed by the authors over the last decade [19].

The rational nullspace computation based techniques allow to solve important applications as the solution of FDP or FDIP. A main feature of rational nullspace based techniques is a full flexibility in addresssing different aspects of these problems, like computing least order detectors, checking existence conditions, computing the achievable fault signature, or employing updating techniques to design a bank of detectors to solve the FDIP. The underlying computations extensively use orthogonal similarity transformations to perform the important computational steps, as for example, to determine a proper nullspace basis or to check the existence conditions of a solution. In contrast, methods based on polynomial nullspace computations are less flexible, and involve computational detours, which are highly questionable from a numerical point of view.

An interesting result of our studies is that although using simple proper rational bases leads to a straightforward solution of the FDP with least order detectors, the computation of the simple basis is not actually necessary. Since we need to compute only linear combinations of simple basis vectors when solving the FDP with least order detector, this computation can be directly performed starting with a minimal proper basis which can be obtained using exclusively orthogonal pencil manipulations. The linear combinations of basis vectors up to a given McMillan degree can be computed using numerical algorithms based on minimal cover techniques. This aspect is highly relevant 
for implementing robust and efficient numerical software as those available in a recent Fault Detection Toolbox for Matlab [23].

\section{Appendix A Computation of minimal dynamic covers}

The computational problem which we solve in this section is the following: given a descriptor pair $(A-\lambda E, B)$ with $A, E \in \mathbb{R}^{n \times n}, B \in \mathbb{R}^{n \times m}$, and $B$ partitioned as $B=\left[\begin{array}{ll}B_{1} & B_{2}\end{array}\right]$ with $B_{1} \in \mathbb{R}^{n \times m_{1}}, B_{2} \in \mathbb{R}^{n \times m_{2}}$, determine the matrix $F \in \mathbb{R}^{m_{2} \times n}$ such that the pair $\left(A+B_{2} F-\lambda E, B_{1}\right)$ is maximally uncontrollable (i.e., $A+B_{2} F-\lambda E$ has maximal number of uncontrollable eigenvalues). A dual problem to be solved in Section 5 deals with an observable pair $(A-\lambda E, C)$ with nonsingular $E$ and with a $C$ matrix partitioned as

$$
C=\left[\begin{array}{l}
C_{1} \\
C_{2}
\end{array}\right]
$$

In this case, a matrix $K$ is sought such that the pair $\left(A+K C_{2}-\lambda E, C_{1}\right)$ is maximally unobservable. For convenience and in agreement with the assumptions of the problem to be solved in Section 5 , we will describe a computational method which is suitable for a controllable pair $(A-\lambda E, B)$ with nonsingular $E$. However, such an algorithm can be immediately applied to solve the above dual problem by applying it to the controllable pair $\left(A^{T}-\lambda E^{T}, C^{T}\right)$ to determine $K^{T}$.

The problem to determine $F$ which makes the pair $\left(A+B_{2} F-\lambda E, B_{1}\right)$ maximally uncontrollable is equivalent [30] to compute a subspace $\mathcal{V}$ of least possible dimension satisfying

$$
\left(\bar{A}+\bar{B}_{2} F\right) \mathcal{V} \subset \mathcal{V}, \quad \operatorname{span}\left(\bar{B}_{1}\right) \subset \mathcal{V},
$$

where $\bar{A}=E^{-1} A, \bar{B}_{1}=E^{-1} B_{1}$, and $\bar{B}_{2}=E^{-1} B_{2}$. This subspace is the least order $\left(\bar{A}, \bar{B}_{2}\right)$-invariant subspace which contains span $\left(\bar{B}_{1}\right)[30]$. The condition (44) can be rewritten as

$$
\bar{A} \mathcal{V} \subset \mathcal{V}+\operatorname{span}\left(\bar{B}_{2}\right), \quad \operatorname{span}\left(\bar{B}_{1}\right) \subset \mathcal{V},
$$

which is the condition defining the subspace $\mathcal{V}$ as a Type I dynamic cover [8].

In this appendix we describe a computational method for determining minimal dynamic covers, which relies on the reduction of the descriptor pair $\left(A-\lambda E,\left[B_{1}, B_{2}\right]\right)$ to a particular condensed form, for which the solution of the problem (i.e., the choice of appropriate $F$ ) is simple. This reduction is performed in two stages. The first stage is an orthogonal reduction which represents a particular instance of the descriptor controllability staircase procedure of [15] applied to the descriptor pair $\left(A-\lambda E,\left[B_{1}, B_{2}\right]\right)$. This procedure can be interpreted as a generalized orthogonal variant of the basis selection approach underlying the determination of Type I minimal covers in [8]. In 
the second stage, additional zero blocks are generated in the reduced matrices using non-orthogonal transformations. With additional blocks zeroed via a specially chosen $F$, the least order $\left(\bar{A}, \bar{B}_{2}\right)$-invariant subspace containing span $\left(\bar{B}_{1}\right)$ can be identified as the linear span of the leading columns of the resulting right transformation matrix. In what follows we present in detail these two stages as well as the determination of $F$.

\section{Stage I: Special Controllability Staircase Algorithm}

0 . Compute an orthogonal matrix $Q$ such that $Q^{T} E$ is upper triangular; compute $A \leftarrow Q^{T} A, E \leftarrow Q^{T} E, B_{1} \leftarrow Q^{T} B_{1}, B_{2} \leftarrow Q^{T} B_{2}$.

1. Set $j=1, r=0, k=2, \nu_{1}^{(0)}=m_{1}, \nu_{2}^{(0)}=m_{2}, A^{(0)}=A, E^{(0)}=E$, $B_{1}^{(0)}=B_{1}, B_{2}^{(0)}=B_{2}, Z=I_{n}$.

2. Compute an orthogonal matrix $W_{1}$ such that

$$
W_{1}^{T}\left[B_{1}^{(j-1)} \mid B_{2}^{(j-1)}\right]:=\left[\begin{array}{c|c}
A_{k-1, k-3} & A_{k-1, k-2} \\
0 & A_{k, k-2} \\
0 & 0
\end{array}\right] \begin{gathered}
\nu_{1}^{(j)} \\
\nu_{2}^{(j)} \\
\nu_{1}^{(j-1)} \\
\nu_{2}^{(j-1)}
\end{gathered}
$$

with $A_{k-1, k-3}$ and $A_{k, k-2}$ full row rank matrices; compute an orthogonal matrix $U_{1}$ such that $W_{1}^{T} E^{(j-1)} U_{1}$ is upper triangular.

3. Compute and partition

$$
\begin{aligned}
& W_{1}^{T} A^{(j-1)} U_{1}:=\left[\begin{array}{ccc}
A_{k-1, k-1} & A_{k-1, k} & A_{k-1, k+1} \\
A_{k, k-1} & A_{k, k} & A_{k, k+1} \\
B_{1}^{(j)} & B_{2}^{(j)} & A^{(j)}
\end{array}\right] \nu_{\rho}^{\nu_{1}^{(j)}} \\
& \nu_{1}^{(j)} \quad \nu_{2}^{(j)} \quad \rho \\
& W_{1}^{T} E^{(j-1)} U_{1}:=\left[\begin{array}{ccc}
E_{k-1, k-1} & E_{k-1, k} & E_{k-1, k+1} \\
O & E_{k, k} & E_{k, k+1} \\
O & O & E^{(j)}
\end{array}\right] \nu_{\rho}^{\nu_{1}^{(j)}} \nu_{\rho}^{(j)}
\end{aligned}
$$

4. For $i=1, \ldots, k-2$ compute and partition

$$
\begin{aligned}
& A_{i, k-1} U_{1}:=\left[\begin{array}{lll}
A_{i, k-1} & A_{i, k} & A_{i, k+1}
\end{array}\right] \\
& \nu_{1}^{(j)} \quad \nu_{2}^{(j)} \quad \rho \\
& E_{i, k-1} U_{1}:=\left[\begin{array}{lll}
E_{i, k-1} & E_{i, k} & E_{i, k+1}
\end{array}\right] \\
& \nu_{1}^{(j)} \quad \nu_{2}^{(j)} \quad \rho
\end{aligned}
$$

5. $Q \leftarrow Q \operatorname{diag}\left(I_{r}, W_{1}\right), Z \leftarrow Z \operatorname{diag}\left(I_{r}, U_{1}\right)$.

6. If $\nu_{1}^{(j)}=0$ then $\ell=j-1$ and Exit.

7. $r \leftarrow r+\nu_{1}^{(j)}+\nu_{2}^{(j)}$; if $\rho=0$ then $\ell=j$ and Exit;

else, $j \leftarrow j+1, k \leftarrow k+2$, and go to Step 2 . 
At the end of this algorithm $\widehat{A}-\lambda \widehat{E}:=Q^{T}(A-\lambda E) Z, \widehat{B}:=Q^{T} B, \widehat{E}$ is upper triangular, and the pair $(\widehat{A}, \widehat{B})$ is in a special staircase form. For example, for $\ell=3$ and $r<n,[\widehat{B} \widehat{A}]$ and $\widehat{E}$ have similarly block partitioned forms

$$
\begin{gathered}
{[\widehat{B} \mid \widehat{A}]=\left[\begin{array}{cc|ccccccc}
A_{1,-1} & A_{1,0} & A_{11} & A_{12} & A_{13} & A_{14} & A_{15} & A_{16} & A_{17} \\
O & A_{2,0} & A_{21} & A_{22} & A_{23} & A_{24} & A_{25} & A_{26} & A_{27} \\
O & O & A_{31} & A_{32} & A_{33} & A_{34} & A_{35} & A_{36} & A_{37} \\
O & O & O & A_{42} & A_{43} & A_{44} & A_{45} & A_{46} & A_{47} \\
O & O & O & O & A_{53} & A_{54} & A_{55} & A_{56} & A_{57} \\
O & O & O & O & O & A_{64} & A_{65} & A_{66} & A_{67} \\
O & O & O & O & O & O & O & A_{76} & A_{77}
\end{array}\right]} \\
\widehat{E}=\left[\begin{array}{cccccc}
E_{11} & E_{12} & \cdots & E_{17} & E_{18} \\
O & E_{22} & \cdots & E_{26} & E_{27} \\
\vdots & \vdots & \ddots & \vdots & \vdots \\
O & O & \cdots & E_{66} & E_{67} \\
O & O & \cdots & O & E_{77}
\end{array}\right]
\end{gathered}
$$

In the special staircase form $[\widehat{B} \widehat{A}], A_{2 j-1,2 j-3} \in \mathbb{R}^{\nu_{1}^{(j)} \times \nu_{1}^{(j-1)}}$ and $A_{2 j, 2 j-2} \in$ $\mathbb{R}^{\nu_{2}^{(j)} \times \nu_{2}^{(j-1)}}$ are full row rank matrices for $j=1, \ldots, \ell$. The trailing row blocks of $[\widehat{B} \widehat{A}]$ and $\widehat{E}$ are empty if $r=n$. In the case when $r<n$, the trailing diagonal blocks $A_{2 \ell+1,2 \ell+1}, E_{2 \ell+1,2 \ell+1} \in \mathbb{R}^{(n-r) \times(n-r)}$, and the pair $\left(A_{2 \ell+1,2 \ell+1}-\lambda E_{2 \ell+1,2 \ell+1}, A_{2 \ell+1,2 \ell}\right)$ is controllable.

In the second reduction stage we use non-orthogonal upper triangular left and right transformation matrices $W$ and $U$, respectively, to annihilate the minimum number of blocks in $\widehat{A}$ and $\widehat{E}$ which allows to solve the minimum cover problem. Assume $W$ and $U$ have block structures identical to $\widehat{E}$. The following procedure exploits the full rank of submatrices $A_{2 j, 2 j-2}$ and $E_{2 j-1,2 j-1}$ to introduce zero blocks in the block row $2 j$ of $\widehat{A}$ and block column $2 j-1$ of $\widehat{E}$, respectively.

\section{Stage II: Special reduction for Type I Covers}

Set $W=I_{n}, U=I_{n}$.

for $k=\ell, \ell-1, \ldots, 2$

Comment. Annihilate blocks $A_{2 k, 2 j-1}$, for $j=k, k+1, \ldots, \ell$.

for $j=k, k+1, \ldots, \ell$

Compute $U_{2 k-2,2 j-1}$ such that $A_{2 k, 2 k-2} U_{2 k-2,2 j-1}+A_{2 k, 2 j-1}=0$.

$A_{i, 2 j-1} \leftarrow A_{i, 2 j-1}+A_{i, 2 k-2} U_{2 k-2,2 j-1}, i=1,2, \ldots, 2 k$.

$E_{i, 2 j-1} \leftarrow E_{i, 2 j-1}+E_{i, 2 k-2} U_{2 k-2,2 j-1}, i=1,2, \ldots, 2 k-2$.

end

Comment. Annihilate blocks $E_{2 k-2,2 j-1}$, for $j=k, k+1, \ldots, \ell$.

for $j=k, k+1, \ldots, \ell$

Compute $W_{2 k-2,2 j-1}$ such that $W_{2 k-2,2 j-1} E_{2 j-1,2 j-1}+E_{2 k-2,2 j-1}=0$. 


$$
\begin{aligned}
& A_{2 k-2, i} \leftarrow A_{2 k-2, i}+W_{2 k-2,2 j-1} A_{2 j-1, i}, i=2 j-2,2 j-1, \ldots, 2 \ell . \\
& E_{2 k-2, i} \leftarrow E_{2 k-2, i}+W_{2 k-2,2 j-1} E_{2 j-1, i}, i=2 j, 2 j+1, \ldots, 2 \ell .
\end{aligned}
$$

end

end

For the considered example, this algorithm introduces the following zero blocks: $A_{65}, E_{45}, A_{43}, A_{45}, E_{23}, E_{25}$ (in this order).

Let $\widetilde{A}:=W \widehat{A} U, \widetilde{E}:=W \widehat{E} U$, and $\widetilde{B}=\left[\widetilde{B}_{1} \widetilde{B}_{2}\right]:=W \widehat{B}$ be the system matrices resulted at the end of Stage II. Define also the feedback matrix $\widetilde{F} \in \mathbb{R}^{m_{2} \times n}$ partitioned column-wise compatibly with $\widehat{A}$

$$
\widetilde{F}=\left[\begin{array}{llllll}
F_{1} & O & F_{3} & \cdots & O & F_{2 \ell-1} O
\end{array}\right]
$$

where $F_{2 j-1} \in \mathbb{R}^{m_{2} \times \nu_{1}^{(j)}}$ are such that $A_{2,0} F_{2 j-1}+A_{2,2 j-1}=0$ for $j=1, \ldots, \ell$.

For the considered example, we achieved with the above cjhoice of $F$ that

$$
\begin{aligned}
\widetilde{A}+\widetilde{B}_{2} \widetilde{F} & =\left[\begin{array}{ccccccc}
\bar{A}_{11} & A_{12} & \bar{A}_{13} & A_{14} & \bar{A}_{15} & A_{16} & A_{17} \\
O & \bar{A}_{22} & O & \bar{A}_{24} & O & \bar{A}_{26} & \bar{A}_{27} \\
A_{31} & A_{32} & \bar{A}_{33} & A_{34} & \bar{A}_{35} & A_{36} & A_{37} \\
O & A_{42} & O & \bar{A}_{44} & O & \bar{A}_{46} & \bar{A}_{47} \\
O & O & A_{53} & A_{54} & \bar{A}_{55} & A_{56} & A_{57} \\
O & O & O & A_{64} & O & A_{66} & A_{67} \\
O & O & O & O & O & A_{76} & A_{77}
\end{array}\right], \\
\widetilde{E} & =\left[\begin{array}{ccccccc}
E_{11} & E_{12} & \bar{E}_{13} & E_{14} & \bar{E}_{15} & E_{16} & E_{17} \\
O & E_{22} & O & \bar{E}_{24} & O & \bar{E}_{26} & \bar{E}_{27} \\
O & O & E_{33} & E_{34} & E_{35} & E_{36} & E_{37} \\
O & O & O & E_{44} & O & \bar{E}_{46} & \bar{E}_{47} \\
O & O & O & O & E_{55} & E_{56} & E_{57} \\
O & O & O & O & O & E_{66} & E_{67} \\
O & O & O & O & O & O & E_{77}
\end{array}\right]
\end{aligned}
$$

where the elements with bars have been modified after Stage I.

Consider now the permutation matrix defined by

$$
P=\left[\begin{array}{cc|cc|c|cc|c}
I_{\nu_{1}^{(1)}} & O & O & O & \cdots & O & O & O \\
\hline O & O & I_{\nu_{1}^{(2)}} & O & \cdots & O & O & O \\
\hline \vdots & \vdots & \vdots & \vdots & \ddots & \vdots & \vdots & \vdots \\
\hline O & O & O & O & \cdots & I_{\nu_{1}^{(\ell)}} & O & O \\
\hline O & I_{\nu_{2}^{(1)}} & O & O & \cdots & O & O & O \\
\hline O & O & O & I_{\nu_{2}^{(2)}} & \cdots & O & O & O \\
\hline \vdots & \vdots & \vdots & \vdots & \ddots & \vdots & \vdots & \vdots \\
\hline O & O & O & O & \cdots & O & I_{\nu_{2}^{(\ell)}} & O \\
\hline O & O & O & O & \cdots & O & O & I_{n-r}
\end{array}\right]
$$


If we define $L=P W Q^{T}, V=Z U P^{T}$ and $F=\widetilde{F} V^{-1}$, then overall we achieved that

$$
L\left(A+B_{2} F-\lambda E\right) V=\left[\begin{array}{c|c}
\breve{A}_{1}-\lambda \breve{E}_{1} & * \\
\hline O & \breve{A}_{2}-\lambda \breve{E}_{2}
\end{array}\right], \quad L\left[B_{1} \mid B_{2}\right]=\left[\begin{array}{c}
\breve{B}_{1} * \\
\hline O \\
\hline O \breve{B}_{2}
\end{array}\right]
$$

where, by construction, the pairs $\left(\breve{A}_{1}-\lambda \breve{E}_{1}, \breve{B}_{1}\right)$ and $\left(\breve{A}_{2}-\lambda \breve{E}_{2}, \breve{B}_{2}\right)$ are in controllable staircase form. Thus, by the above choice of $F$, we made $n_{2}:=$ $\sum_{i=1}^{\ell} \nu_{2}^{(i)}$ of the $n$ eigenvalues of the $A+B_{2} F-\lambda E$ uncontrollable via $B_{1}$. It is straightforward to show that the matrix $V_{1}$ formed from the the first $n_{1}:=\sum_{i=1}^{\ell} \nu_{1}^{(i)}$ columns of $V$, satisfies

$$
\bar{A} V_{1}=V_{1} \breve{E}_{1}^{-1} \breve{A}_{1}-\bar{B}_{2} F V_{1}, \quad \bar{B}_{1}=V_{1} \breve{E}_{1}^{-1} \breve{B}_{1}
$$

Thus, according to (45), $\mathcal{V}:=\operatorname{span}\left(V_{1}\right)$ is a dynamic cover of Type $I$ of dimension $n_{1}$. It can be shown using the results of [8] that the resulting Type $I$ dynamic cover $\mathcal{V}$ has minimum dimension.

For the considered example, we obtained the controllable staircase forms

$$
\begin{aligned}
& {\left[\breve{B}_{1} \mid \breve{A}_{1}-\lambda \breve{E}_{1}\right]=\left[\begin{array}{c|ccc}
A_{1,-1} & \bar{A}_{11}-\lambda E_{11} & \bar{A}_{13}-\lambda \bar{E}_{13} & \bar{A}_{15}-\lambda \bar{E}_{15} \\
O & A_{31} & \bar{A}_{33}-\lambda E_{33} & \bar{A}_{35}-\lambda \bar{E}_{35} \\
O & O & A_{53} & \bar{A}_{55}-\lambda E_{55}
\end{array}\right]} \\
& {\left[\breve{B}_{2} \mid \breve{A}_{2}-\lambda \breve{E}_{2}\right]=\left[\begin{array}{c|cccc}
A_{2,0} & \bar{A}_{22}-\lambda E_{22} & \bar{A}_{24}-\lambda \bar{E}_{24} & \bar{A}_{26}-\lambda \bar{E}_{26} & \bar{A}_{27}-\lambda \bar{E}_{27} \\
O & A_{42} & \bar{A}_{44}-\lambda E_{44} & \bar{A}_{46}-\lambda E_{46} & \bar{A}_{47}-\lambda \bar{E}_{47} \\
O & O & A_{64} & A_{66}-\lambda E_{66} & A_{67}-\lambda E_{67} \\
O & O & O & A_{76} & \bar{A}_{77}-\lambda E_{77}
\end{array}\right]}
\end{aligned}
$$

The Stage I reduction of system matrices to the special controllability form can be performed by using exclusively orthogonal similarity transformations. It can be shown that the computed condensed matrices $\widehat{A}, \widehat{E}$, and $\widehat{B}$ are exact for matrices which are nearby to the original matrices $A, E$, and $B$, respectively. Thus this part of the reduction is numerically backward stable. When implementing the algorithm, the row compressions are usually performed using rank revealing QR-factorizations with column pivoting.

To achieve an $O\left(n^{3}\right)$ computational complexity in Stage I reduction, it is essential to perform the row compressions simultaneously with maintaining the upper triangular shape of $E$ during reductions. The basic computational technique, described in details in [15], consists in employing elementary Givens transformations from left to introduce zero elements in the rows of $B$, while applying from right appropriate Givens transformations to annihilate the generated nonzero subdiagonal elements in $E$. By performing the rank revealing QR-decomposition in this way (involving also column permutations), we can show that the overall worst-case computational complexity of the special staircase algorithm is $O\left(n^{3}\right)$. Note that for solving the problem in Section 5, the 
accumulation of $Z$ is not even necessary, since all right transformations can be directly applied to a third matrix (e.g., a system output matrix $C$ ).

The computations at Stage II reduction to determine a basis for the minimal dynamic cover and the computation of the feedback matrix $F$ involve the solution of many, generally overdetermined, linear equations. For the computation of the basis for $\mathcal{V}$ it is important to estimate the condition numbers of the overall transformation matrices. This can be done by computing $\|V\|_{F}^{2}=\|U\|_{F}^{2}$ and $\|L\|_{F}^{2}=\|W\|_{F}^{2}$ as estimations of the corresponding condition numbers. If these norms are relatively small (e.g., $\leq 10000)$ then practically there is no danger for a significant loss of accuracy due to nonorthogonal reductions. On contrary, large values of these norms provide a clear hint of potential accuracy losses. In practice, it suffices only to look at the largest magnitudes of elements of $W$ and $U$ used at Stage II to obtain equivalent information. For the computation of $F$, condition numbers for solving the underlying equations can be also easily estimated. A large norm of $F$ is an indication of possible accuracy losses. For the Stage II reduction, a simple operation count is possible by assuming all blocks $1 \times 1$ and this indicates a computational complexity of $O\left(n^{3}\right)$.

\section{References}

1. E.N. Antoniou, A.I.G. Vardulakis, and S. Vologiannidis. Numerical computation of minimal polynomial bases: A generalized resultant approach. Lin. Alg. \& Appl., 405:264-278, 2005.

2. Th.G.J. Beelen. New Algorithms for Computing the Kronecker structure of a Pencil with Applications to Systems and Control Theory. Ph. D. Thesis, Eindhoven University of Technology, 1987.

3. X. Ding and P. M. Frank. Frequency domain approach and threshold selector for robust model-based fault detection and isolation. In Proc. of IFAC Symposium SAFEPROCESS'1991, Baden-Baden, Germany, 1991.

4. G. D. Forney. Minimal bases of rational vector spaces with applications to multivariable linear systems. SIAM J. Control, 13:493-520, 1975.

5. E. Frisk and M. Nyberg. A minimal polynomial basis solution to residual generation for fault diagnosis in linear systems. Automatica, 37:1417-1424, 2001.

6. J. Gertler. Fault Detection and Diagnosis in Engineering Systems. Marcel Dekker, New York, 1998.

7. T. Kailath. Linear Systems. Prentice Hall, Englewood Cliffs, N.J., 1980.

8. G. Kimura. Geometric structure of observers for linear feedback control laws. IEEE Trans. Automat. Control, 22:846-855, 1977.

9. D. G. Luenberger. Observers for multivariable systems. IEEE Trans. Automat. Control, 11:190 - 197, 1966.

10. A. S. Morse. Minimal solutions to transfer matrix equations. IEEE Trans. Automat. Control, 21:131-133, 1976.

11. M. Nyberg. Criterions for detectability and strong detectability of faults in linear systems. Int. J. Control, 75:490-501, 2002.

12. P. Van Dooren. The generalized eigenstructure problem in linear systems theory. IEEE Trans. Automat. Control, 26:111-129, 1981. 
13. A. I. G. Vardulakis and N. Karcanias. Proper and stable, minimal MacMillan degrees bases of rational vector spaces. IEEE Trans. Automat. Control, 29:1118 $-1120,1984$.

14. A. Varga. Numerically stable algorithm for standard controllability form determination. Electron. Lett., 17:74-75, 1981.

15. A. Varga. Computation of irreducible generalized state-space realizations. $K y$ bernetika, 26:89-106, 1990.

16. A. Varga. On stabilization of descriptor systems. Systems \& Control Letters, 24:133-138, 1995.

17. A. Varga. Computation of Kronecker-like forms of a system pencil: Applications, algorithms and software. In Proc. CACSD'96 Symposium, Dearborn, MI, pages 77-82, 1996.

18. A. Varga. Computation of coprime factorizations of rational matrices. Lin. Alg. \& Appl., 271:83-115, 1998.

19. A. Varga. A Descriptor Systems toolbox for Matlab. In Proc. CACSD'2000 Symposium, Anchorage, Alaska, 2000.

20. A. Varga. On computing least order fault detectors using rational nullspace bases. In Proc. of IFAC Symp. SAFEPROCESS'2003, Washington D.C., 2003.

21. A. Varga. New computational approach for the design of fault detection and isolation filters. In M. Voicu, editor, Advances in Automatic Control, volume 754 of The Kluwer International Series in Engineering and Computer Science, pages 367-381. Kluwer Academic Publishers, 2004.

22. A. Varga. Reliable algorithms for computing minimal dynamic covers for descriptor systems. In Proc. of MTNS'04, Leuven, Belgium, 2004.

23. A. Varga. A Fault Detection toolbox for Matlab. In Proc. of CACSD'06, Munich, Germany, 2006.

24. A. Varga. On designing least order residual generators for fault detection and isolation. In Proc. 16th Internat. Conf. on Control Systems and Computer Science, Bucharest, Romania, pages 323-330, 2007.

25. A. Varga. General computational approach for optimal fault detection. In Proc. SAFEPROCESS'2009, Barcelona, Spain, 2009.

26. A. Varga. The nullspace method - a unifying paradigm to fault detection. In Proc. CDC'2009, Shanghai, China, 2009.

27. A. Varga. On computing achievable fault signatures. In Proc. SAFEPROCESS'2009, Barcelona, Spain, 2009.

28. G. Verghese, P. Van Dooren, and T. Kailath. Properties of the system matrix of a generalized state-space system. Int. J. Control, 30:235-243, 1979.

29. M. E. Warren and A. E. Eckberg. On the dimension of controllability subspaces: A characterization via polynomial matrices and Kronecker invariants. SIAM J. Contr., 13:434-445, 1975.

30. W. M. Wonham and A. S. Morse. Feedback invariants of linear multivariable systems. Automatica, 8:93-100, 1972.

31. Z. Yuan, G. C. Vansteenkiste, and C. Y. Wen. Improving the observer-based FDI design for efficient fault isolation. Int. J. Control, 68(1):197-218, 1997.

32. K. Zhou, J. C. Doyle, and K. Glover. Robust and Optimal Control. Prentice Hall, 1996. 